\title{
INCREASED ACCURACY OF THE NAKAGAMI $M$ PARAMETER MEASUREMENT METHOD BASED ON SPACE DIVERSITY RECEPTION
}

UDC (621.396.67:004.6)

\section{Vladeta Milenković, Dragan Denić, Aleksandar Jocić}

University of Niš, Faculty of Electronic Engineering, Department of Measurement, Niš, Republic of Serbia

\begin{abstract}
In this paper we will present increased accuracy measurement method for the Nakagami-m m parameter estimation, based on implementation of space diversity reception. Parameter $m$ will be estimated in real time, based on the obtained values of first, second and third order moments of signal, measured by a proposed system. The obtained results will show an increase of accuracy obtained by using this method over the results obtained by using a standard measurement procedure, so it creates a potential to apply this measurement method for estimation of fading parameters in various propagation environments.
\end{abstract}

Key words: Antenna diversity reception, Data acquisition, Data processing, Parameter estimation

\section{INTRODUCTION}

The Nakagami- $m$ distribution is considered as one of the most important fading models among all the statistical ones that have been proposed to characterize the random envelope due to multipath propagation in wireless communications. The Nakagami- $m$ fading model describes multipath scattering with large delay-time spreads, and different clusters of reflected waves, and provides good fits to collected data in indoor and outdoor wireless environments [1]. The Nakagami- $m$ fading model exploits the Nakagami- $m$ probability density function (PDF) for the envelope of received signal, which is written in the terms of two parameters: the scale parameter and shape parameter. The shape parameter is more important and is called the fading parameter or $m$-parameter. In order to use the Nakagami- $m$ distribution to model a given set of empirical fading data, one must determine or estimate the value of $m$ from these measured data. Accurate estimation of the $m$ parameter is for example

Received October 31, 2017

Corresponding author: Vladeta Milenković

Faculty of Electronical Engineering, Aleksandra Medvedeva 14, 18000 Niš, Republic of Serbia

E-mail: vladeta.milenkovic@ @etico-group.com 
required by the receiver for optimal reception of signals in the Nakagami- $m$ fading. An estimated value of the $m$ parameter in such a case is feedback to the transmitter side, so that the transmission is adapted by taking account of the channel information [2]. The most wellknown methods used for the estimation of the Nakagami fading parameter $m$, are the maximum likelihood (ML) estimation and moment-based estimators. However, the ML estimation procedure reduces to a problem of solving a complicated transcendental equation written in the termsof a logarithmic function and a gamma function [3]. Moment based estimation has been widely considered in technical literature, recently [4-5]. A method for estimation of parameter $m$ based on both integer sample momens and non-integer sample moments, along with simulation study was proposed in [6].

Various drawbacks occur within wireless transmission. These drawbacks that affect a desired signal could be identified, mathematically modelled and minimized by corresponding methods obtained by observing adequate measurements [7-10] and processing by recorded data series [11]. In this paper we will present the moment-based $m$ parameter estimation method with increased accuracy, based on the measurement method performed by applying of space diversity reception. Parameter $m$ will be estimated in real time, based on the obtained values of first, second and third order moments of signal, measured by the proposed system.

\section{SUBJECT AND METHODS}

The probability density function (PDF) for a Nakagami-m distributed channel can be expressed as [1]:

$$
p_{r}(r)=\frac{2 r^{2 m-1} m^{m}}{\Gamma(m) \Omega^{m}} \exp \left(-\frac{m r^{2}}{\Omega}\right)
$$

where the channel amplitude $\mathrm{R} \geq 0, \Omega=\mathrm{E}\left(\mathrm{R}^{2}\right)$ is average fading signal power, $\mathrm{E}()$ is the expectation operator, and $\Gamma()$ is gamma function [12]. Above, $m$ is the Nakagami fading parameter which determines the severity of the fading. The value for $m$ ranges between $1 / 2$ and $\infty$. When $m \rightarrow \infty$ fading channel converges a static channel. As special cases, Nakagami-m includes Rayleigh distribution when $\mathrm{m}=1$, and one-sided Gaussian distribution for $m=1 / 2$. This basically means that, if $m<1$, the Nakagami- $m$ distributed fading is more severe than Rayleigh fading, and for values of $m>1$, the fading circumstances are less severe. For the values of $m>1$, the Nakagami- $m$ distribution closely approximates the Rician distribution, and the parameters $m$ and the Rician factor $K$ (which determines the severity of the Rician fading) can be mapped via parameter $m$.

From the previous text, it is evident that for a reasonable estimation and measurement of Nakagami- $m$ fading parameters, it is necessary to determine its moments. For the $n$-th order moment of the Nakagami- $m$ random variable $r$ we have:

$$
m_{n}=\overline{r^{n}}=\frac{1}{\Gamma(m)}\left(\sqrt{\frac{\Omega}{m}}\right)^{n} \Gamma\left(\frac{2 m+n}{2}\right)
$$


It was shown in [14] that the Nakagami parameter $m$ can be determined in the function of first order moment $\mathrm{m}_{1}$, second order moment $\mathrm{m}_{2}$ and third order moment $\mathrm{m}_{3}$, as:

$$
m=\frac{1}{2}\left(\frac{m_{1} m_{2}}{m_{3}-m_{1} m_{2}}\right)
$$

Let us now also consider diversity reception influence on the relative error in the process of the $n$-th moment determination. As already known, with MRC combining, the received signals are co-phased, each signal is amplified appropriately for optimal combining, and the resulting signals are added so an envelope at the MRC output $R$ is given with [1]:

$$
R=\sqrt{\sum_{i=1}^{L} R_{i}^{2}}
$$

After $L$-branch MRC with Rayleigh-fading signals, the resulting signal is Nakagami-m with parameter $m=L$. MRC combining of Nakagami-m fading signals in $L$ branches gives a Nakagami signal with shape factor $m L$. The resulting pdf of the instantaneous envelope $r$, at the output of the MRC combiner is given in the literature in the form of [13]:

$$
p_{M R C}(r)=\frac{2 r^{2 m L-1}(m L)^{m L}}{\Gamma(m L) \Omega^{m L}} \exp \left(-\frac{m r^{2}}{\Omega}\right)
$$

while the $n$-th order moment of the random variable $r$ at the output of the combiner is given in the form of:

$$
\overline{r_{M R C}^{n}}=\frac{1}{\Gamma(m L)}\left(\sqrt{\frac{\Omega}{m}}\right)^{n} \Gamma\left(\frac{2 m L+n}{2}\right)
$$

In [14] theoretical bounds to the relative error expressions are obtained for the cases with and without MRC diversity combining at the receiver. It is shown that a lower relative error should be expected when MRC diversity is present at the reception.

The measurement system with applied space diversity for Nakagami $m$ parameter estimation has been shown at figure 1. It consists of two receiver antennas leaded at two spectrum analyzers, that are driven by PC, each through RS232 serial communication port. Data acquisition has been performed by LabVIEW software and followed by software for space diversity technique realization and corresponding parameter estimation $\left(m_{1}, m_{2}, m_{3}, m \ldots\right)$.

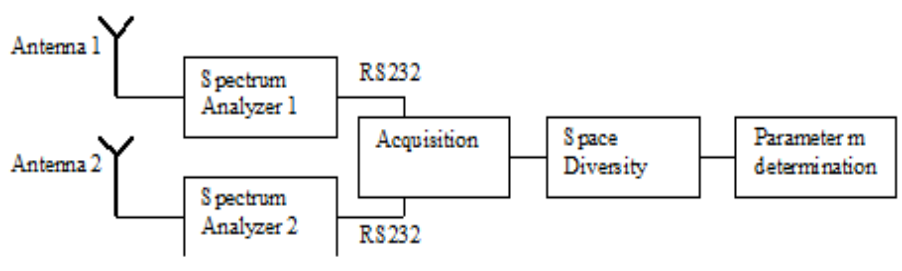

Fig. 1 System block scheme. 
A measured fading signal, whose parameters have been estimated, is constantly present, since being the signal used for the purpose of regulating the railway traffic. The signal is vertically polarized, frequency-modulated with a carrier frequency of $\mathrm{f}_{0}=467.88 \mathrm{MHz}$. At the reception two 7-element $8.5 \mathrm{~dB}$ gain YAGI antennas, which are adapted for operating in the UHF frequency range of $390-475 \mathrm{MHz}$ bandwidth have been used. The antennas are connected with measuring devices via an $\mathrm{N}$-connector coaxial cable.

Two spectrum analyzers, which precisely measure the power level of the received signal at a specified frequency, in corresponding time instants are used as measuring devices. Spectrum analyzers are driven by PC, and PC works as a master device, which can set corresponding value at its serial communication port and trigger acquisition of data from each spectrum analyzer. In that manner data acquisition is performed on PC, and values of the power level signal are measured at exactly the right frequency and in relatively short time intervals (max 20 times per second).

Software for acquisition performs two major functions: 1) At a certain period of time, it triggers spectrum analyzers, by sending an appropriate sequence, to set the corresponding values on them 2) Take the data from spectral analyzers via serial communication ports, and memorize it in a separate file. Depending on the type of diversity technique that is implemented (MRC, EGC...), series of samples input signals $\mathrm{x}_{1 \mathrm{i}}$ and $\mathrm{x}_{2 \mathrm{i}}$ are processed by corresponding algorithm and novel series of samples $y_{\text {out }}$ is formed, which now represents the output of diversity system. Further, special software calculates the first, second and third order moments for observed series of data [11]. Now, since dependence of fading severity parameter estimation $\mathrm{m}$ depends of the measured values of the sample series order moments, then accurate calculation of order moments must be provided in both cases, with and without diversity implemented. Measurement system realization has been presented at Fig. 2., while LabView implementation of measuring system was presented at Fig. 3.

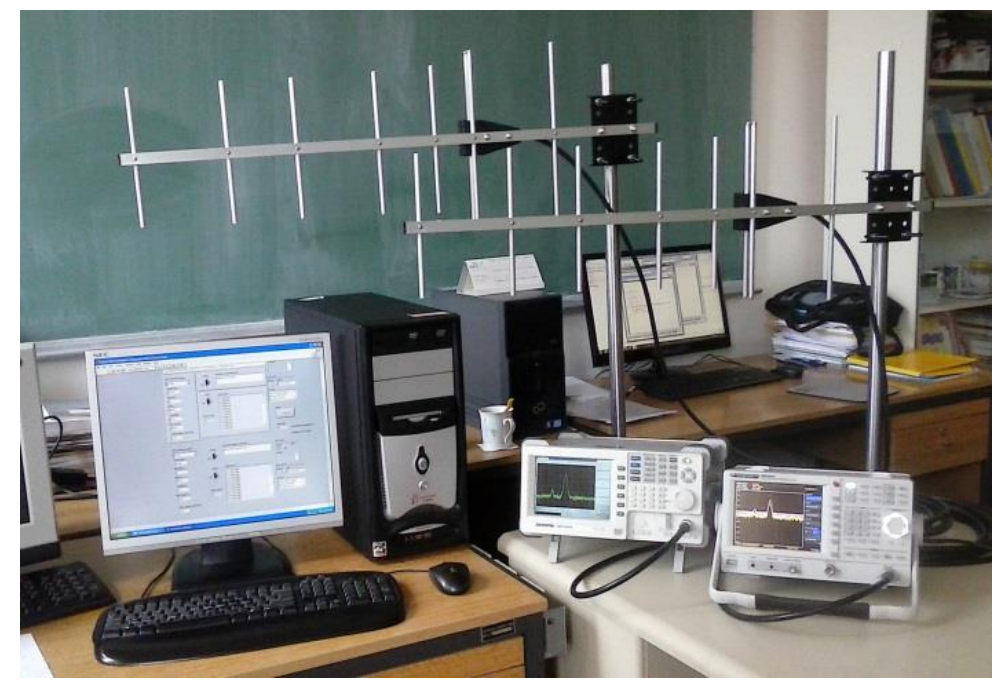

Fig. 2 Measurement system 


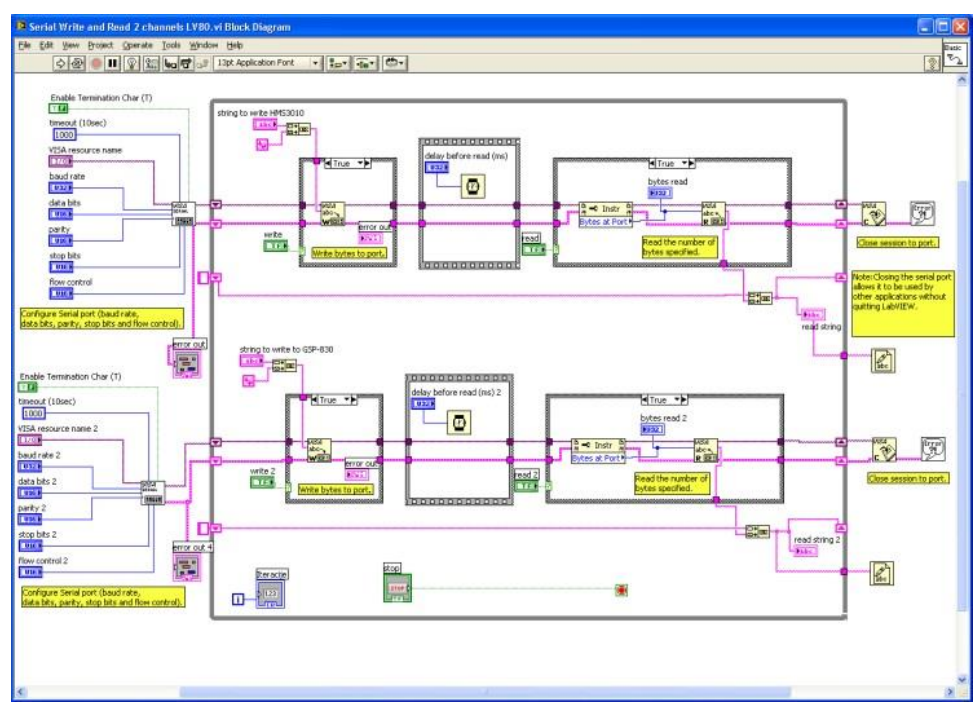

Fig. 3 LabView block-scheme implementation of measuring system

\section{RESULTS}

After practical realization of measurement system based on the applied space diversity we acquired real data and showed how sensitivity of parameter $m$ estimation on the estimation error of sample moments indeed reduces when diversity is applied. Estimation accuracy is presented and analyzed in the function of the size of acquired data.

Series of 100,000 samples, sampled at 10sample/second, are acquired by a corresponding measurement system in order to provide a reliable analysis of Nakagami- $m$ parameter [15]. Different intervals of this sample series $(0-20000, \ldots, 0-100000)$, are divided into frames of 1000 samples. For each frame of samples, first, second and third order sample moments values are determined, first when observing only the data collected from a single antenna.

Based on their values, the parameter $m$ value was estimated according to (3). Then, first, second and third order sample moments values, $m_{l a v}, m_{2 a v}$ and $m_{3 a v}$ are determined, and based on their values the parameter $m_{a v}$ value was estimated when the whole series of 100000 collected samples, was observed. Now aberrations from these values [11] are calculated observing values obtained for each frame according to:

$$
\begin{aligned}
& \Delta m_{1}=\sqrt{\frac{1}{N} \sum_{j=1}^{N}\left(m_{1 j}-m_{1 a v}\right)^{2}} \\
& \Delta m=\sqrt{\frac{1}{N} \sum_{j=1}^{N}\left(m_{j}-m_{a v}\right)^{2}}
\end{aligned}
$$

where $N$ denotes number of frames that series has been divided to. Sensitivity of parameter $m$ estimation error on the estimation error of sample moment of first order $\mathrm{m} 1$ can be now expressed as: 


$$
\delta=\frac{\Delta m}{\Delta m_{1}}
$$

As already mentioned in [14], it is expected that by applying the MRC diversity system, the effects of measurement errors in the determination of the values of moment of the first order $\mathrm{m}_{1}$ are incorporated in the estimation of the Nakagami parameter $m$ are at most two times lower (theoretical bound) than the effects of the measurement error of the first order moment $\mathrm{m}_{1}$ incorporated in the estimation of Nakagami parameter $m$ for the case when no diversity technique has been applied, i.e.:

$$
\left(\frac{\Delta m^{\text {out })}}{\Delta m_{1}{ }^{\text {out })}}\right) /\left(\frac{\Delta m^{(n)}}{\Delta m_{1}{ }^{(n)}}\right)=\frac{\delta_{\text {out }}}{\delta_{n}}=\frac{1}{2}
$$

where $n=1,2$, stands for a number of the antenna (the input to the diversity system), while out - the output of the diversity of the system. In table 1, are shown the effects of measurement errors of the first order $\mathrm{m}_{1}$ on the estimation of the Nakagami parameter $m$ for different intervals of this sample series $(0-20000, \ldots, 0-100000)$, with intervals divided into frames of 2000 samples, for both cases with and without diversity model implementation.

As it can be seen from the Table 1, when diversity method is applied, accuracy increases, i.e. sensitivity of parameter $m$ estimation error on the estimation error of sample moment of first order $m_{1}$ decreases when samples of combined signal are observed. As evident this sensitivity measure is lower in the whole range of observed samples, which means that more accurate estimation of parameter $m$ should be obtained by observing combined acquired data.

The parameter $m$ values obtained based on combined acquired data are shown in Figure 4. Since we are observing fast fading on corresponding carrier frequency, random process that changes in time, the values of parameter $m$ are estimated for a corresponding series of samples. The major advantage of this approach is its generality in fading characterization.

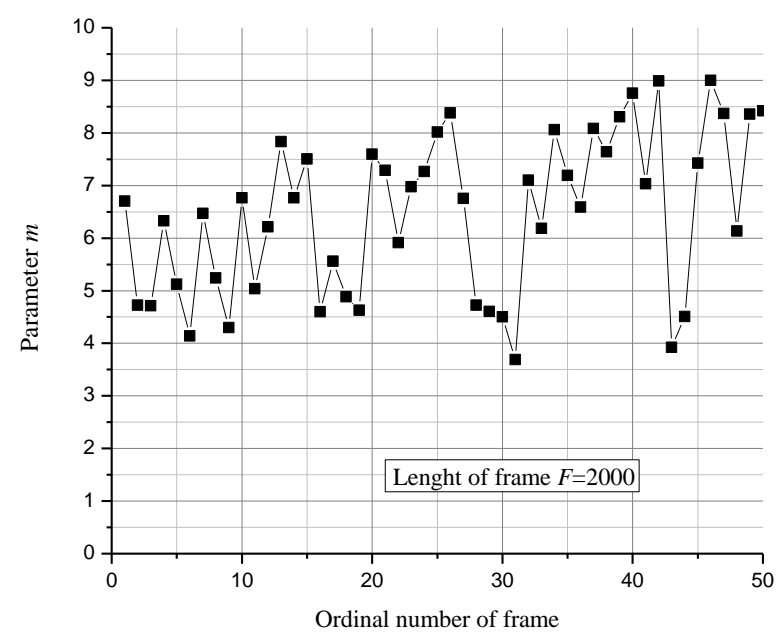

Fig. 4 Values of parameter $m$ obtained by proposed measurement method 
Namely, since the Nakagami-m fading model includes in itself other fading models, that can be obtained by setting corresponding values to the Nakagami-m model parameters, by estimating parameter $m$ we are at the same time estimating the parameters of other fading models. For example in the area of higher values of a parameter $\mathrm{m}$, from its estimated value, we can easily obtain the value of parameter $K$ which characterizes Rician fading distribution model with strong line-of-sight (LOS) component, by using relation between above mentioned parameters as $m=(1+K)^{2} /(1+2 K)$. In a similar manner when estimating smaller values of the parameter $m$, the fading process can be modelled with Hoyt model, whose determination parameter $q$ can be derived by using its well-known relation to estimated $m$ value.

Table 1 Effects of measurement errors of the first order $m_{1}$ on the estimation of the Nakagami parameter $m$ for different intervals of this sample series with and without diversity model implementation

\begin{tabular}{cccccc}
\hline Number of samples & $0-20 \mathrm{~K}$ & $0-40 \mathrm{~K}$ & $0-60 \mathrm{~K}$ & $0-80 \mathrm{~K}$ & $0-100 \mathrm{~K}$ \\
\hline$\delta_{1}$ & 4.161 & 7.234 & 1.01 & $1.22 \mathrm{E}-3$ & 1.41 \\
& $\mathrm{E}-4$ & $\mathrm{E}-4$ & $\mathrm{E}-3$ & & E-3 \\
$\delta_{\text {out }}$ & 2.53 & 4.84 & 6.969 & 8.91 & 1.029 \\
& $\mathrm{E}-4$ & $\mathrm{E}-4$ & $\mathrm{E}-4$ & $\mathrm{E}-4$ & $\mathrm{E}-4$ \\
$\delta_{\text {out } /} \delta_{\mathrm{n}}$ & 0.61 & 0.67 & 0.69 & 0.73 & 0.73 \\
\hline
\end{tabular}

\section{CONCLUSION}

We have presented the increased accuracy measurement method for the Nakagami- $m \mathrm{~m}$ parameter estimation, based on implementation of combining acquired data. By applying the presented method, the parameter $m$ can be estimated in real time, and fading propagation environment can be efficiently characterized. Further, capitalizing on the measurement and estimation characteristics, wireless link parameters can be adapted in order to meet predetermined grade-of-service and quality-of-service values at the reception, by using minimal transmission power and minimizing channel capacity.

Acknowledgement: This work was supported by the Serbian Ministry of Science (grant \# III42009).

\section{REFERENCES}

[1] S. Panic, et.al. Fading and interference mitigation in wireless communications. CRC Press, 2014.

[2] E. Belding-Royer, A. Khaldoun, G. Pujolle, Mobile and Wireless Communication Networks, Springer.

[3] J. Cheng, N. C. Beaulieu, N. C. "Maximum-Likelihood based estimation of the Nakagami m parameter", IEEE Commun. Lett., vol. 5, no. 3, pp. 101-103, 2001.

[4] J. Cheng, N. C. Beaulieu, "Estimation of Ricean and Nakagami distribution parameters using noisy samples", in Proc. IEEE International Conf. on Commn. (ICCS04), Paris, France, June 2004, pp. 562-566.

[5] J. Cheng, N. C. Beaulieu, "Novel Nakagami-m parameter estimator for noisy channel samples", IEEE Communication Letters, vol. 9, no. 5, pp. 417-419, 2002.

[6] N. Wang, X. Song, J. Cheng "Generalized Method of Moments Estimation of the Nakagami-m Fading Parameter", IEEE Transactions on Wireless Communications, vol. 11, no. 9, pp. 3316 - 3325, 2012. 
[7] F. Luan, Y. Zhang, L. Xiao, C. Zhou, S. Zhou, "Fading Characteristics of Wireless Channel on HighSpeed Railway in Hilly Terrain Scenario", International Journal of Antennas and Propagation, Article ID $378407,1-9,2013$.

[8] M. Malović, Lj. Brajović, Z. Mišković, T. Šekara, "Simultaneity Analysis In A Wireless Sensor Network, Metrology and Measurement Systems”, vol. 22, no. 2, pp. 275-288, 2015.

[9] P. Varlamos, L. Heretakis, P. Papakanellos, P. Trakadas, C. Capsalis, "Measurements and simulation for a joint non-Gaussian fast-fading model in indoor-propagation environments", Microwawe and Optical Technology Letters, vol. 45, no. 6,pp. 515-519, 2005.

[10] L. Angrisani, F. Cennamo, G. Scarpato, R. Moriello, "Prototype of a Dsp-Based Instrument for InService Wireless Transmitter Power Measurement”, Metrology and Measurement Systems, vol. 21, no. 4, pp. 669-708, 2014.

[11] J. Mandel, The Statistical Analysis of Experimental Data. New York, Interscience-Wiley Publishers, out of print; corrected and reprinted, New York, Dover Publishers, 1964

[12] I. S. Gradshteyn, I. M. Ryzhik, Table of Integrals, Series, and Products, Academic Press, 2007.

[13] V. Milentijević, V., et. al., "Relative measurement error analysis in the process of the Nakagami-m fading parameter estimation”, Serbian Journal of Electrical Engineering, vol. 8, no. 3, pp. 341-349.

[14] D. Denic, et. al., "The Method for Fading Measurement in Outdoor Propagation Environments and its Improvement by applying Diversity Technique", in Proc., XI International SAUM Conference on Systems, Automatic Control and Measurements Niš, Serbia, November 14th-16th, 2012, pp. 88-91.

[15] Y. Cho, J. Kim, W. Yang, C. Kang, MIMO-OFDM Wireless Communications with MATLAB, Singapore, John Wiley \& Sons, 2010. 